\title{
Minimal required exposures reveal the primacy of awareness in human face perception
}

\author{
Renzo C. Lanfranco ${ }^{1,2^{*}}$, Andrés Canales-Johnson ${ }^{3,4}$, Hugh Rabagliati ${ }^{1}$, Axel Cleeremans ${ }^{5 \dagger}$, \& \\ David Carmel ${ }^{6,1^{*}+}$
}

1Department of Psychology, University of Edinburgh, Edinburgh, United Kingdom; ${ }^{2}$ Department of Neuroscience, Karolinska Institutet, Stockholm, Sweden; ${ }^{3}$ Department of Psychology, University of Cambridge, Cambridge, United Kingdom; ${ }^{4}$ Vicerrectoría de Investigación y Posgrado, Universidad Católica del Maule, Talca, Chile; ${ }^{5}$ Center for Research in Cognition and Neurosciences, Université Libre de Bruxelles, Brussels, Belgium; 'SChool of Psychology, Victoria University of Wellington, Wellington, New Zealand.

*Corresponding authors: Dr. Renzo Lanfranco, Department of Neuroscience, Karolinska Institutet, Biomedicum, D4, Solnavägen 9, 171 65, Stockholm, Sweden. Email: renzo.lanfranco@ki.se, and Dr. David Carmel, School of Psychology, EA 618, Easterfield Building, Gate 3, Kelburn Parade, Wellington, New Zealand. Email: david.carmel@vuw.ac.nz

'Shared senior authorship: Axel Cleeremans and David Carmel.

\begin{abstract}
The human visual system is capable of detecting extremely small light signals, as little as one single photon. But what is the visual system's minimal exposure threshold to detect meaningful stimuli? Due to hardware limitations, studies examining fast visual processing typically present stimuli for suprathreshold durations and disrupt processing with a mask. Here, we employed a newly developed tachistoscope that enables sub-millisecond presentations. Combining measures of perceptual sensitivity and recordings of neural activity, we find that both the holistic configuration and the emotional expression of a human face have the same minimal required exposure as the minimal required exposure for faces to reach awareness. These findings indicate that conscious awareness is required for processing meaningful visual information.
\end{abstract}


The human visual system is so sensitive that observers can report experiencing light signals as minimal as a single photon $(1,2)$. But characterising the minimal stimulation that is required for humans to experience meaningful stimuli, like human faces, has proven controversial. For theories of vision and awareness, the functional and neural distinction between stimulation that is subliminal versus supraliminal is of critical consequence. Theories of consciousness, for example, differ as to whether they propose an allor-nothing approach to awareness, where subliminal stimuli receive minimal processing while stimuli that are even just above threshold receive global processing, versus propose that even subliminal stimuli can be processed in higher-order fashion. Meanwhile, data from brief visual presentations has also been at-issue for theories of visual object recognition, and debates as to whether this process is staged or cascaded. Is there a minimal stimulation that, if exceeded, makes all aspects of such stimuli detectable? Or are there different minimal stimulation thresholds for the detection of different stimulus characteristics? Here, we used faces, a stimulus category that conveys a wealth of meaningful information that plays a major role in human life. Furthermore, face processing is associated with a host of behavioural $(3,4)$ and neural markers (57).

However, current methods for establishing minimal stimulation all have well-recognised limitations. Critically, due to hardware limitations, it has not been possible to present complex images at briefer exposures than $\sim 16 \mathrm{~ms}$, which is a sufficiently long exposure for visual detection and identification to occur (810). Trying to circumvent this limitation, researchers have employed numerous masking and interocular suppression techniques that have enabled them to interrupt visual processing and prevent conscious awareness. But since it is impossible to determine the extent of this interruption, these techniques cannot provide with minimal exposure thresholds of perceptual processes. Additionally, it is unclear whether the minimal exposure thresholds that they provide are due to differences in the time necessary to process relevant stimulus properties, or in the degree to which these techniques affect the processing of those stimulus properties $(11,12)$. Ultimately, because it has not been determined what specific aspects of visual processing masking and suppression techniques interrupt, it is also not possible to determine whether findings using masked and unmasked meaningful stimuli can be compared.

To determine the minimal exposure thresholds of meaningful stimuli, we showed human participants face images in different orientations and with different emotional expressions using a newly developed LCD tachistoscope that enables extremely brief visual presentations with sub-millisecond precision ( 2 microsec; see supplementary materials for technical details). By employing psychophysical techniques, we measured detection and emotion identification, and how sensitive participants' subjective reports were to their own detection performance of faces (Experiments 1 and 2). In addition, we searched for electroencephalography (EEG) markers of face and emotion processing, and of conscious access (Experiments 3 and 4).

A group of 32 healthy participants were presented with an upright or inverted face 
bioRxiv preprint doi: https://doi.org/10.1101/2021.10.06.462913; this version posted October 15, 2021. The copyright holder for this preprint (which was not certified by peer review) is the author/funder, who has granted bioRxiv a license to display the preprint in perpetuity. It is made available under aCC-BY-NC 4.0 International license.

image and its scrambled counterpart, one on each side, for extremely brief predetermined exposures (Fig. 1). Participants reported the location of the intact face (left or right) and identified its expression (emotional or non-emotional) with a single keypress; then, they reported how clear was their visual experience of the intact face.

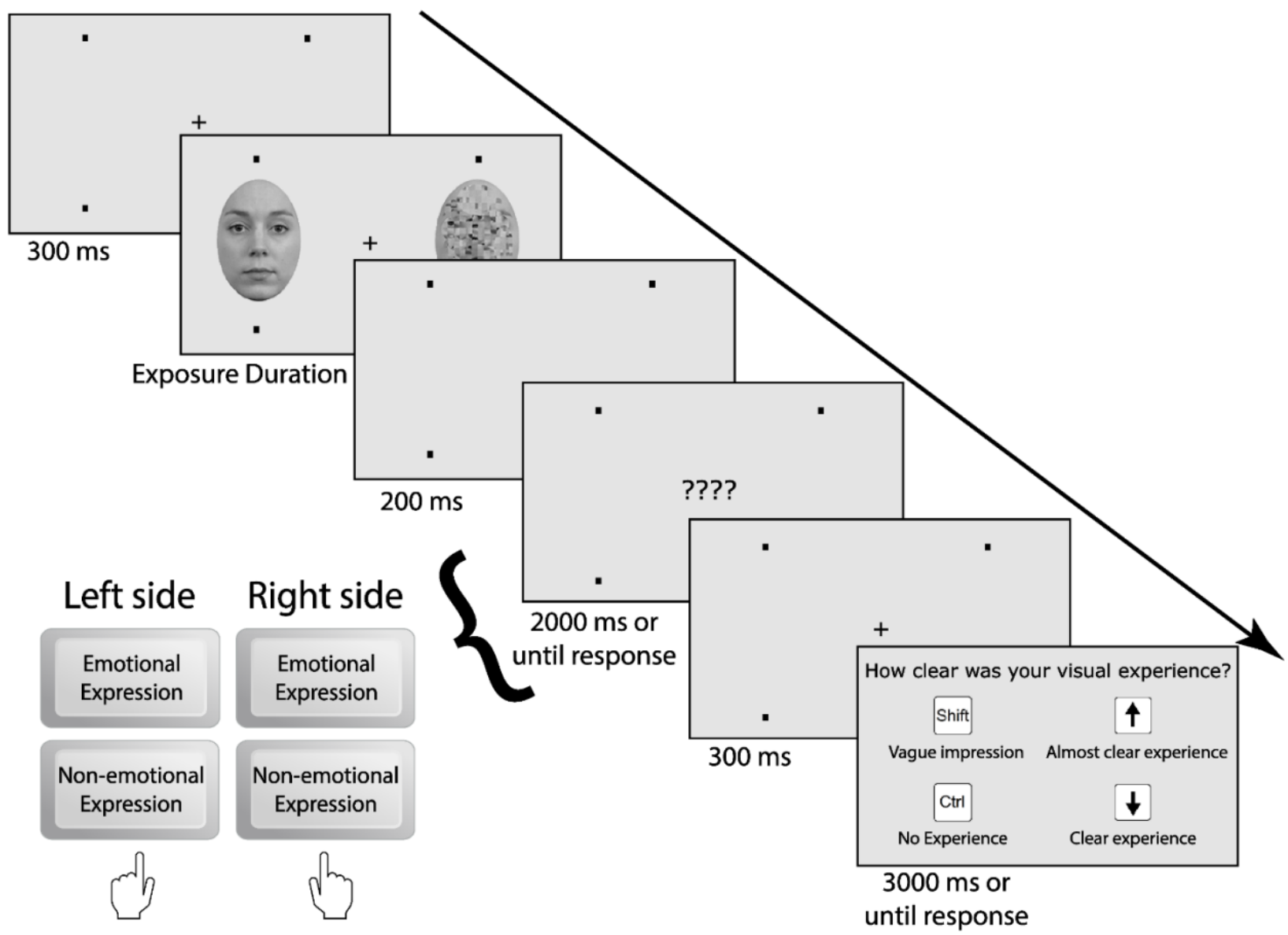

Fig. 1. Schematic description of a trial. Stimuli were presented for one of seven possible exposure durations (0.8 - $6.2 \mathrm{~ms}$, equally spaced on a linear scale). After stimulus offset, participants judged the location of the intact face (left or right) and its expression (emotional or non-emotional) by pressing one key. Next, they judged the clarity of their visual experience. Face stimuli were selected from the Radboud Faces Database (RaFD; 13).

An increase in exposure from 0.8 to 6.2 $\mathrm{ms}$ led to a significant increase in sensitivity to the location of the intact face $\left(F_{(4.90,151.98)}=15.331, p<.001, \eta p^{2}=.331\right)$, from chance performance to high sensitivity, and with an advantage for upright faces over inverted faces $\left(F_{(1,31)}=\right.$ 34.198, $\left.p<.001, \eta p^{2}=.530\right)$. This advantage for faces with their holistic properties intact (upright faces) - also known as the face-inversion effect (FIE) - is regarded as a perceptual index of holistic processing $(4,6,13,14)$. This FIE interacted with the increase in exposure; the FIE arose with $4.4 \mathrm{~ms}$ of exposure $\left(F_{(4.90,151.98)}=15.331, p<.001, \eta p^{2}=.331\right)$, indicating this as the minimal exposure threshold of holistic processing (Fig. 2A). However, while emotional faces did not exhibit a significant advantage over 
bioRxiv preprint doi: https://doi.org/10.1101/2021.10.06.462913; this version posted October 15, 2021. The copyright holder for this preprint (which was not certified by peer review) is the author/funder, who has granted bioRxiv a license to display the preprint in perpetuity. It is made available under aCC-BY-NC 4.0 International license.

neutral faces in location sensitivity, we did find a FIE in emotion identification: identification sensitivity increased with exposure

$$
\left(F_{(4.22,130.93)}=12.89, p<\right.
$$
$\left..001, \eta \mathrm{p}^{2}=.294\right)$, with a FIE $\left(F_{(1,31)}=\right.$ $\left.19.54, p<.001, \eta p^{2}=.387\right)$ and a robust interaction between orientation and exposure

$\left(F_{(4.75,147.15)}=3.12, p=\right.$ $\left..012, \eta \mathrm{p}^{2}=.091\right)$. This FIE of emotion identification arose with $5.3 \mathrm{~ms}$ of exposure $(t(209)=3.563, p=.041, d=$ 0.630), (Fig. 2B). Together, these results indicate that holistic face processing precedes emotion identification.

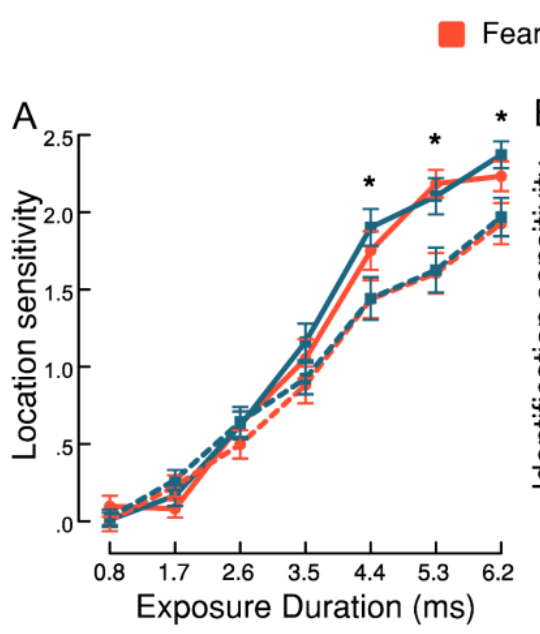

Fearful Neutral

- Upright _- Inverted
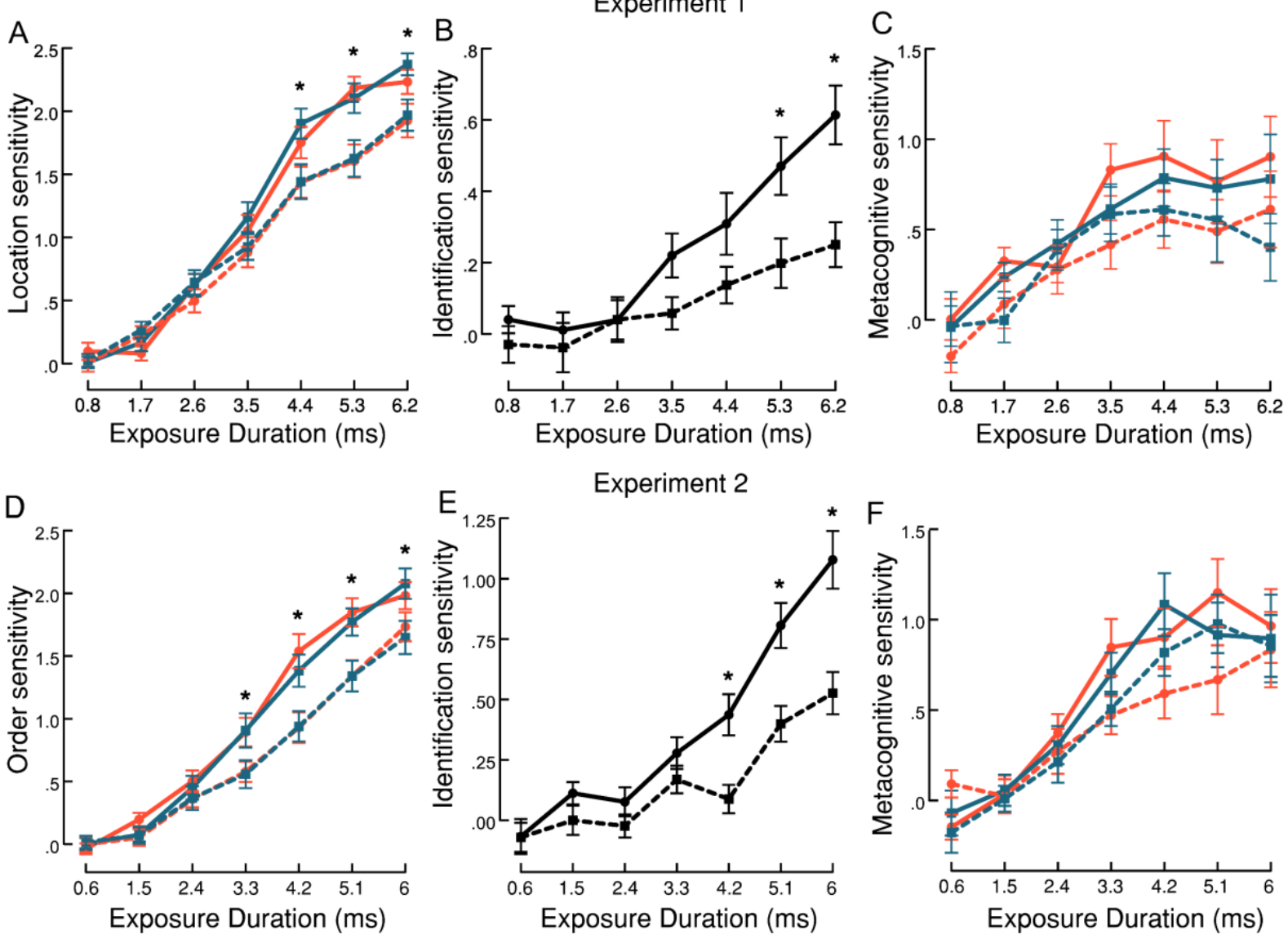

Fig. 2. Holistic face processing precedes expression identification. (Top) Peripheral vision discrimination. (A) Location sensitivity increased with increasing exposure durations, departing from chance performance around $2 \mathrm{~ms}$. A significant advantage for upright faces over inverted faces is present from $4.4 \mathrm{~ms}$ of exposure. (B) Identification sensitivity for expression increased with increasing exposure durations. A significant advantage in expression identification for upright over inverted faces arose from $5.3 \mathrm{~ms}$ of exposure. (C) Awareness-based metacognitive sensitivity increased with exposure duration. Upright faces enjoyed higher sensitivity than inverted faces overall, suggesting that upright faces reached awareness earlier than inverted faces. (Bottom) Foveal vision discrimination. (D) Order sensitivity, (E) Expression identification sensitivity, and (C) Awarenessbased metacognitive sensitivity showed the same pattern of results found with peripheral vision discrimination. Error bars represent standard error of the mean (SEM). 
The sensitivity of participants' subjective reports to their detection performance (metacognitive sensitivity) also exhibited a FIE - sensitivity scores increased as exposures increased $\quad\left(F_{(3.69,114.38)}=\right.$ $\left.12.922, p<.001, \eta p^{2}=.294\right)$, with an advantage of upright over inverted faces overall $\quad\left(F_{(1,31)}=6.475, p=.016, \eta \mathrm{p}^{2}=\right.$ .173), (Fig. 2C).

But are these results specific to peripheral vision? Another group of 32 participants were presented with intact and scrambled face images on the centre of the screen, but on different presentation orders; they had to discriminate the intact face's presentation order. They exhibited the same pattern of results described above, thus supporting the generalisability of these findings (Fig. 2D-F). Moreover, these results cannot be attributed to afterimage processing (see supplementary materials), ruling out the possibility that retinal images may have confounded the detection of meaningful images.

But does neural emotion processing have these same minimal exposure thresholds? A new group of 32 participants performed a very similar task to that described above (peripheral discrimination; Fig.1) but this time we also recorded their EEG data (see supplementary materials). We first extracted two classic event-related potential (ERP) markers of emotion processing: the early posterior negativity (EPN; 15) and the late positive potential (LPP; 16). EPN was able to discriminate between emotional and neutral expressions $\left(F_{(1,31)}=6.042, p=.02, \eta p^{2}=\right.$ .163). The interaction between expression and exposure was robust $\left(F_{(1.90,58.86)}=\right.$
8.675, $\left.p<.001, \eta \mathrm{p}^{2}=.219\right)$ :

EPN's sensitivity to emotional expression arose only at the longest exposure $(6.2 \mathrm{~ms}$; Fig. $3 \mathrm{~A})$, as indexed by a more negative voltage fluctuation associated with emotional expressions $(t(87.6)=$ $-4.009, p=.002, d=-0.709)$. Similarly, LPP showed a robust interaction between expression and exposure $\left(F_{(1.98,61.40)}=\right.$ $\left.9.80, p<.001, \eta p^{2}=.240\right)$ : LPP's sensitivity to emotional expression also arose at the longest exposure (6.2 ms; Fig. 3B), as indexed by a more positive voltage fluctuation associated with emotional expressions $(t(90.3)=4.284, p<.001$, $d=0.757)$. Taken together, ERP markers indicate that the minimal exposure threshold of neural emotion processing is not shorter than $4.4 \mathrm{~ms}$ nor longer than $6.2 \mathrm{~ms}$.

Since ERP analysis may not be sufficiently sensitive to multivariate neural patterns, we also used multivariate pattern analysis (MVPA) to test for differences that ERP analysis may have missed. First, we trained a classifier to decode stimulus location; the classifier decoded intact faces from scrambled ones by $4.4 \mathrm{~ms}$ (Fig. 3C). However, no expression enjoyed better classification performance than the others. Secondly, we trained another classifier to decode fearful, happy, and neutral expressions altogether (multiclass classification) regardless of their presentation location; the classifier decoded between these expressions by $4.4 \mathrm{~ms}$ (Fig. 3D). These results indicate that the minimal exposure threshold of neural processing of face and emotion content is around $4.4 \mathrm{~ms}$. 

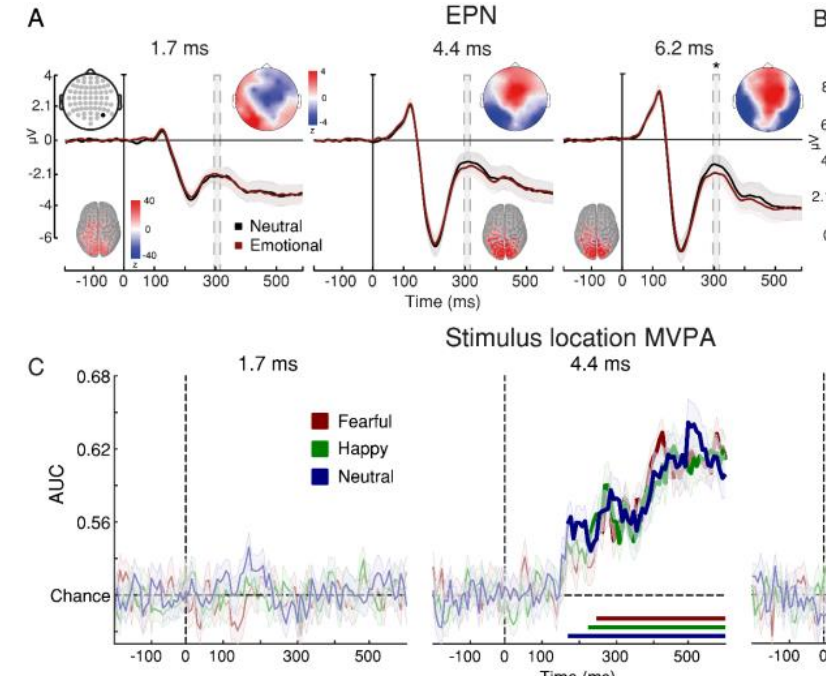

Stimulus location MVPA
$4.4 \mathrm{~ms}$
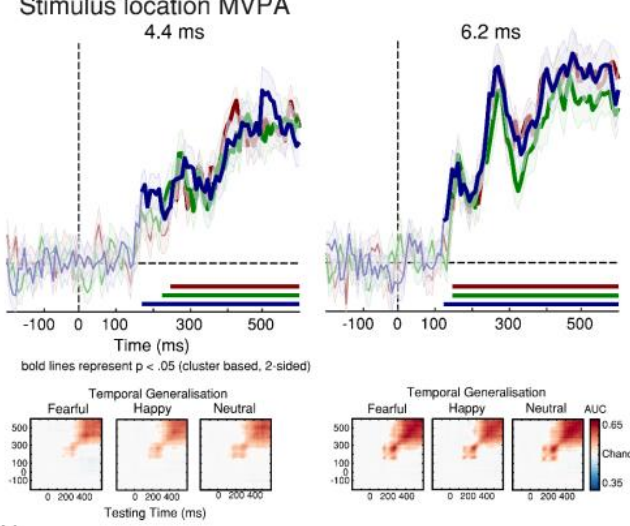

VAN
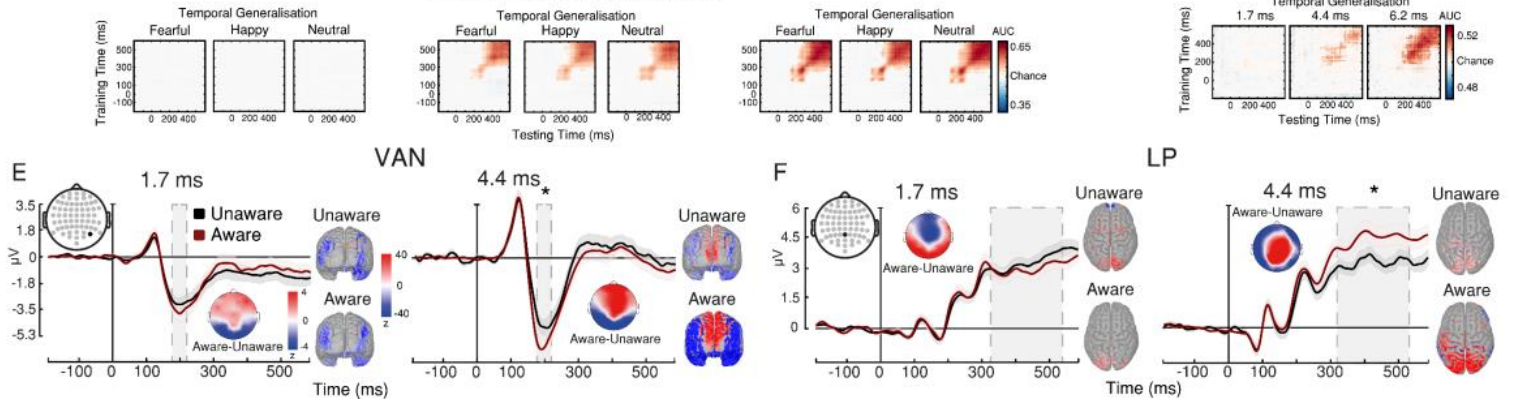

Fig. 3. Emotion processing and conscious access unfold together in the brain. (A) EPN discriminated between emotional and neutral expressions only at the longest exposure, as indexed by a significantly more negative voltage response to emotional expressions. (B) LPP showed equivalent results, as indexed by a significantly more positive voltage response to emotional expressions. Topographic maps represent subtraction of voltage values between emotional and neutral faces. Source estimation of the ERPs at their voltage peaks are visually identified on cortical maps. (C) The MVPA classifier decoded stimulus location with above-chance performance only with 4.4 and $6.2 \mathrm{~ms}$ of exposure. No differences were found between expressions. The temporal generalisation analysis, which tested to what extend the multivariate neural patterns of each training time point ( $\mathrm{Y}$-axis) generalises to testing time points (X-axis) showed broad temporal generalisation with those two exposures. (D) The MVPA classifier decoded the three expressions with above-chance performance only with 4.4 and $6.2 \mathrm{~ms}$ of exposure, with broad temporal generalisation. Both (E) VAN and (F) LP were modulated by awareness reports with $4.4 \mathrm{~ms}$ of exposure. Shaded contours represent SEM.

Does processing meaningful stimuli require conscious access? To determine the minimal exposure threshold of conscious access, we extracted two ERP markers that index conscious awareness: the visual awareness negativity (VAN; 17, 18) and Late Positivity (LP; 19-21). By comparing trials that received a subjective report of visual awareness with trials that received a subjective report of no awareness, we could find the exposure range in which visual information was consciously accessed (see supplementary materials): VAN was sensitive to awareness reports $\left(F_{(1,30)}=17.755, p<\right.$ $\left..001, \eta p^{2}=.372\right)$ and showed an interaction between awareness report and exposure $\quad\left(F_{(1,30)}=10.062, p=\right.$ $\left..003, \eta p^{2}=.251\right)$. VAN's sensitivity to awareness arose by $4.4 \mathrm{~ms}$ of exposure $(t(45.4)=-5.21, p<.001, d=-0.935)$, (Fig. 3E). Similarly, LP was also sensitive to 
awareness reports $\quad\left(F_{(1,30)}=7.737, p=\right.$ $\left..009, \eta \mathrm{p}^{2}=.205\right)$, and showed an interaction between awareness report and exposure $\quad\left(F_{(1,30)}=37.420, p<\right.$ $\left..001, \eta \mathrm{p}^{2}=.555\right)$. LP's sensitivity to awareness also arose by $4.4 \mathrm{~ms}$ of exposure $\quad(t(55)=5.861, p<.001, d=$ 1.053), (Fig. 3F). Thus, ERP markers indicate that the minimal exposure threshold for meaningful stimuli to be consciously accessed is not shorter than 4.4 ms nor longer than $6.2 \mathrm{~ms}$. Our results, therefore, indicate that neural emotion processing and conscious access of faces have the same minimal exposure threshold, which also corresponds to the minimal exposure threshold of holistic face processing.

Holistic processing, emotion processing, and conscious access of faces share the same minimal exposure threshold and therefore may unfold together. However, while the FIE is a classic and widely accepted perceptual index of holistic processing, we wondered whether neural markers of face processing had the same minimal exposure threshold than the FIE. To address this, a new group of 32 participants were presented with a face or object image and its scrambled counterpart, one on each side, for briefer exposures, ranging from 0.8 to $4.288 \mathrm{~ms}$, while their EEG data were collected. The rest of the task remained the same as in Experiment 1. First, we extracted a classic ERP marker of face processing: the N170 $(5,6)$, which is sensitive to face stimuli, discriminating them from object stimuli.
Indeed, we found that the N170's voltage response became more negative with increasing exposures $\quad\left(F_{(1.38,42.92)}=\right.$ $\left.45.681, p<.001, \eta p^{2}=.596\right)$ and showed a robust interaction between stimulus category and exposure $\left(F_{(2.45,75.89)}=\right.$ $\left.6.398, p=.001, \eta p^{2}=.171\right)$, successfully discriminating between face and object stimuli by $4.288 \mathrm{~ms}$ of exposure $(t(114)=$ $-3.467, p=.021, d=-0.613$ ), (Fig. 4A). Moreover, we searched for multivariate differences in neural patterns that ERP analysis may have missed; we did so by training a classifier to decode stimulus location (MVPA). The classifier decoded intact faces from scrambled faces only by $4.288 \mathrm{~ms}$ of exposure (Fig. 4B) and with relatively wide temporal generalisation, thus supporting the N170 findings (also see supplementary materials).

Neural face processing and conscious access unfolded together: VAN was sensitive to awareness reports $\left(F_{(1,30)}=\right.$ $\left.19.612, p<.001, \eta p^{2}=.395\right)$, although in this experiment VAN did not show an interaction between awareness report and exposure (Fig. 4C). Nevertheless, LP was sensitive to awareness reports $\left(F_{(1,30)}=4.826, p=.036, \eta p^{2}=.139\right) \quad$ and showed an interaction between awareness reports and exposure $\left(F_{(2.33,70.03)}=5.393, p=.004, \eta p^{2}=.152\right)$. Crucially, LP's sensitivity to awareness arose by $4.288 \mathrm{~ms}$ of exposure $(t(87.3)=$ 3.683, $p=.011, d=0.661)$. Therefore, both ERPs and MVPA results indicate that neural face processing and conscious access unfold together. 
A $\quad 0.8 \mathrm{~ms}$
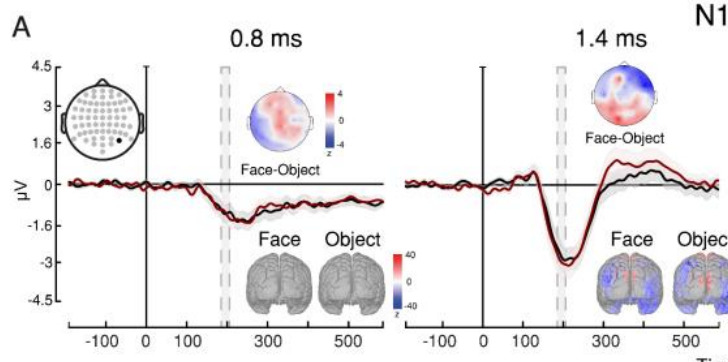

N170

Stimulus location MVPA

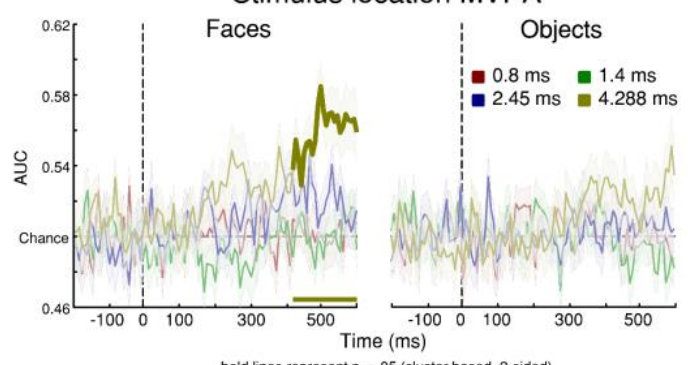

Time (ms)

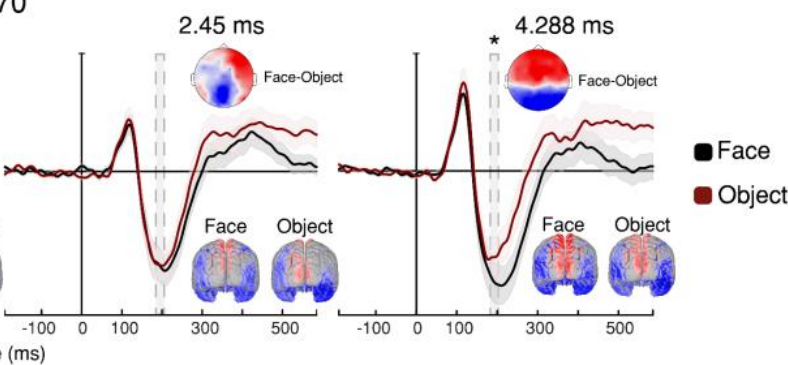

bold lines represent p < . 05 (cluster based, 2-2sided)

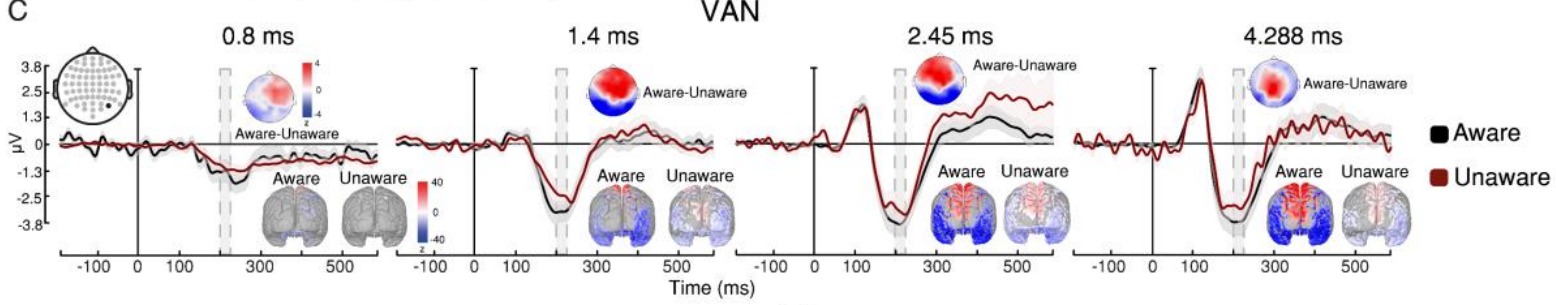

D
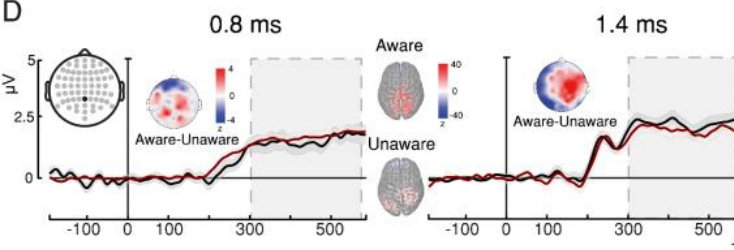

LP

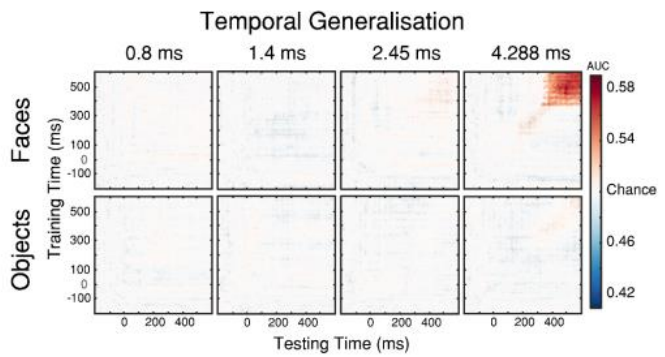

VAN
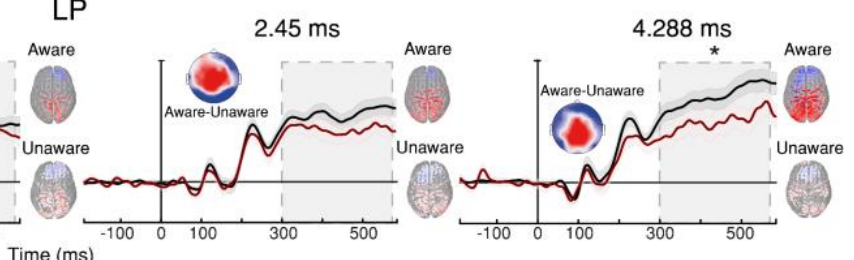

Fig. 4. Face processing and conscious access unfold together in the brain. (A) N170 discriminated between face and object stimuli only with $4.288 \mathrm{~ms}$, as indexed by a significantly more negative voltage response to faces. Topographic maps represent subtraction of voltage values between face and object stimuli. Source estimations of the ERPs at their voltage peaks are visually identified on cortical maps. (B) The MVPA classifier decoded face stimulus location with abovechance performance only with $4.288 \mathrm{~ms}$ of exposure. Object stimulus location was not decoded. The temporal generalisation analysis, which tested to what extend the multivariate neural patterns of each training time point ( $Y$-axis) generalise to all testing time points ( $X$-axis) showed broad temporal generalisation only with 4.288 ms of exposure and only for face stimuli. Both (C)VAN and (D) LP were modulated by awareness reports but only LP showed an interaction between awareness reports and exposure - LP was sensitive to awareness reports only with $4.288 \mathrm{~ms}$ of exposure. Shaded contours represent SEM.

In summary, our findings reveal that different meaningful features of human faces such as holistic configuration and emotional expression have the same minimal exposure threshold as the minimal exposure required for conscious access to unfold. We propose that conscious awareness is required for processing complex features contained in meaningful stimuli, thus questioning past masking and interocular suppression studies that have claimed the existence of unconscious processing of meaningful stimuli (e.g. 22, 12, but see 24, 25). Both 
psychophysical

and

electroencephalographic methods have provided a convergent picture on a minimal exposure threshold that conveys sufficient bottom-up input for meaningful stimuli to enter sensory processing and gain access to awareness. Conscious awareness, therefore, may allow the neural broadcasting $(22,27,28)$ of those visual features that, when integrated, create perceptual meaning.

\section{References}

1. S. Hecht, S. Shlaer, M. H. Pirenne*, Energy at the Threshold of Vision. Science. 93, 585-587 (1941).

2. J. N. Tinsley, M. I. Molodtsov, R. Prevedel, D. Wartmann, J. EspiguléPons, M. Lauwers, A. Vaziri, Direct detection of a single photon by humans. Nat Commun. 7, 12172 (2016).

3. R. K. Yin, Looking at upside-down faces. Journal of Experimental Psychology. 81, 141-145 (1969).

4. M. J. Farah, J. W. Tanaka, H. M. Drain, What causes the face inversion effect? Journal of Experimental Psychology: Human Perception and Performance. 21, 628-634 (1995).

5. S. Bentin, T. Allison, A. Puce, E. Perez, G. McCarthy, Electrophysiological Studies of Face Perception in Humans. Journal of Cognitive Neuroscience. 8 , 551-565 (1996).

6. N. Kanwisher, F. Tong, K. Nakayama, The effect of face inversion on the human fusiform face area. Cognition. 68, B1-B11 (1998).

7. M. Eimer, A. Holmes, Event-related brain potential correlates of emotional face processing. Neuropsychologia. 45, 15-31 (2007).

8. M. Fabre-Thorpe, A. Delorme, C. Marlot, S. Thorpe, A Limit to the Speed of Processing in Ultra-Rapid Visual Categorization of Novel Natural Scenes. Journal of Cognitive Neuroscience. 13, 171-180 (2001).

9. S. Thorpe, D. Fize, C. Marlot, Speed of processing in the human visual system. Nature. 381, 520-522 (1996).

10. G. Rousselet, O. Joubert, M. FabreThorpe, How long to get to the "gist" of real-world natural scenes? Visual Cognition. 12, 852-877 (2005).

11. M. Codispoti, M. Mazzetti, M. M. Bradley, Unmasking emotion: Exposure

duration and emotional engagement. Psychophysiology. 46, 731-738 (2009).

12. M. R. Greene, A. Oliva, The Briefest of Glances: The Time Course of Natural Scene Understanding. Psychological Science (2009) (available at http://journals.sagepub.com/doi/10.11 11/j.1467-9280.2009.02316.x).

13. O. Langner, R. Dotsch, G. Bijlstra, D. H. J. Wigboldus, S. T. Hawk, A. van Knippenberg, Presentation and validation of the Radboud Faces Database. Cognition and Emotion. 24, 1377-1388 (2010).

14. R. I. Goodrich, A. P. Yonelinas, Journal of Experimental Psychology: General, in press, doi:10.1037/xge0000618.

15. G. Yovel, N. Kanwisher, The Neural Basis of the Behavioral Face-Inversion Effect. Current Biology. 15, 2256-2262 (2005).

16. B. Rossion, S. Caharel, ERP evidence for the speed of face categorization in the human brain: Disentangling the contribution of low-level visual cues from face perception. Vision Research. 51, 1297-1311 (2011).

17. Y. Liu, H. Huang, M. McGinnis-Deweese, A. Keil, M. Ding, Neural Substrate of the Late Positive Potential in Emotional Processing. J. Neurosci. 32, $14563-$ 14572 (2012).

18. R. Eklund, S. Wiens, Visual awareness negativity is an early neural correlate of awareness: A preregistered study with two Gabor sizes. Cogn Affect Behav Neurosci. 18, 176-188 (2018).

19. M. Koivisto, S. Grassini, Neural processing around $200 \mathrm{~ms}$ after stimulus-onset correlates with subjective visual awareness. Neuropsychologia. 84, 235-243 (2016).

20. A. Del Cul, S. Baillet, S. Dehaene, Brain Dynamics Underlying the Nonlinear Threshold for Access to Consciousness. PLOS Biology. 5, e260 (2007). 
21. D. Lamy, M. Salti, Y. Bar-Haim, Neural Correlates of Subjective Awareness and Unconscious Processing: An ERP Study. Journal of Cognitive Neuroscience. 21, 1435-1446 (2008).

22. C. Sergent, S. Baillet, S. Dehaene, Timing of the brain events underlying access to consciousness during the attentional blink. Nature Neuroscience. 8, 1391-1400 (2005).

23. E. Yang, D. H. Zald, R. Blake, Fearful expressions gain preferential access to awareness during continuous flash suppression. Emotion. 7, 882-886 (2007).

24. L. Mudrik, A. Breska, D. Lamy, L. Y. Deouell, Integration Without Awareness: Expanding the Limits of Unconscious Processing. Psychol Sci. 22, 764-770 (2011).

25. K. L. H. Gray, W. J. Adams, N. Hedger, K. E. Newton, M. Garner, Faces and awareness: Low-level, not emotional factors determine perceptual dominance. Emotion. 13, 537-544 (2013).

26. N. Biderman, L. Mudrik, Evidence for Implicit-But Not UnconsciousProcessing of Object-Scene Relations. Psychol Sci. 29, 266-277 (2018).

27. C. Sergent, M. Corazzol, G. Labouret, F. Stockart, M. Wexler, J.-R. King, F. Meyniel, D. Pressnitzer, Bifurcation in brain dynamics reveals a signature of conscious processing independent of report. Nature Communications. 12 1149 (2021).

28. S. Dehaene, J.-P. Changeux, L. Naccache, J. Sackur, C. Sergent, Conscious, preconscious, and subliminal processing: a testable taxonomy. Trends in Cognitive Sciences. 10, 204-211 (2006).
Society, a Principal's Go Abroad Fund awarded by the University of Edinburgh, and partially by a postdoctoral fellowship awarded by Karolinska Institutet to Renzo Lanfranco; by a European Research Council (ERC) Advanced Grant (RADICAL ADG-340718) to Axel Cleeremans; by an Economic and Social Research Council (ESRC) Future Research Leaders award (no. ES/L01064X/1) to Hugh Rabagliati; and by a ERC Advanced Grant (XPECT - DLV692739) to David Carmel. The authors thank Simon van Gaal and Robert Mclntosh for their comments and suggestions; Johannes Fahrenfort, Maximilian Bruchmann, Stephen Fleming, Albert De Beir, and Brian Maniscalco for their technical advice; and Dalila Achoui for her technical guidance.

\section{Competing interests}

The authors declare no competing interests.

\section{Acknowledgements}

This work was supported by a PhD studentship awarded by ANID/CONICYT, a Postgraduate Study Visit Scheme grant awarded by the British Psychological 